\title{
Anwendung von Gebirgsklassifikationssystemen im untertägigen Bergbau
}

\author{
Patrick Kirschner und Anja Moser \\ Department Mineral Resources Engineering, Montanuniversität Leoben, Leoben, Österreich \\ Eingegangen 28. April 2017; angenommen 2. Mai 2017; online publiziert 16. Mai 2017
}

Zusammenfassung: Die weit verbreiteten und vielfach angewandten Methoden der Gebirgsklassifikation können herangezogen werden, um die Qualität von Gebirge in Bezug auf dessen Standfestigkeit zu beurteilen. Da die Gebirgsklassifikationsmethoden für unterschiedliche Einsatzgebiete und Gebirgstypen entwickelt wurden, heutzutage jedoch gleichermaßen zur Gebirgsgütebeurteilung herangezogen werden, haben sich einige Schwierigkeiten in der Anwendung ergeben. Weiters fehlen in einigen Fällen konkrete Angaben zur Vorgehensweise bei der Durchführung der Gebirgsklassifikation, insbesondere, wenn die Bedingungen von jenen abweichen, für die die Systeme ursprünglich entwickelt wurden. Somit ist es Ziel dieser am Lehrstuhl für Bergbaukunde, Bergtechnik und Bergwirtschaft angefertigten Bachelorarbeit, die am häufigsten verwendeten Methoden der Gebirgsklassifikation nach Bieniawski (1989), Barton (1974), Laubscher (1990) und Hoek (1994) zu analysieren, die Schwierigkeiten bei der Anwendung aufzuzeigen und in weiterer Folge ein Tool zu entwickeln, das die Anwendung der Verfahren zur Beurteilung der Gebirgsgüte vereinfachen soll. Mit Hilfe dieses Tools soll die Beurteilung von Gebirge auch für Personen mit begrenzter Erfahrung in Bezug auf Gebirgsaufnahmen möglich sein.

Schlüsselwörter: Gebirgsklassifikation, Untertagebergbau

Application of Rock Mass Classification Systems in Underground Mining

Abstract: Rock mass classification systems are widely used for the assessment of the rock mass stability. As a result of the application of the rock mass classification systems in fields for which they were not originally designed, difficulties arise in the use of the systems. Furthermore, specific

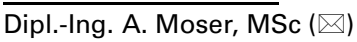

Department Mineral Resources Engineering,

Montanuniversität Leoben,

Franz-Josef-Str. 18,

8700 Leoben, Österreich

anja.moser@unileoben.ac.at indications for the conduction of the rock mass classification in situ are missing in most cases, especially when the application conditions differ from the original development conditions. In the framework of a bachelor thesis at the Chair of Mining Engineering and Mineral Economics the most commonly used rock mass classification systems after Bieniawski (1989), Barton (1974), Laubscher (1990) and Hoek (1994) are analysed and difficulties during the application are highlighted. As a final step a tool to ease the application of these systems is created. Using the developed worksheet, the assessment of the rock mass quality should be possible also for people with limited experience in rock mass documentation.

Keywords: Rock mass classification, Underground mining

\section{Einleitung}

Auslegung und Planung von untertägigen Hohlräumen muss in Bezug auf die vorherrschenden Gebirgsbedingungen erfolgen, um gleichzeitig produktives und sicheres Gewinnen von Rohstoffen zu gewährleisten. Somit müssen die Gebirgsbedingungen beurteilt werden, um alle Tätigkeiten auf die Eigenschaften des Gebirges abzustimmen. Dies stellt eine große Herausforderung dar, da durch Laboruntersuchungen an Gesteinsproben aus dem Gebirge dessen Eigenschaften nur bedingt quantifiziert werden können und In-situ-Versuche mit erheblichem Kostenund Zeitaufwand verbunden sind. Die Quantifizierung der Gebirgseigenschaften ist jedoch von beträchtlicher Bedeutung, da für Standfestigkeitsberechnungen repräsentative mechanische Eigenschaften des Gebirges benötigt werden. Um diese Problematik zu umgehen, wurden indirekte Methoden zur Bestimmung der Gebirgseigenschaften und zur Abschätzung der Gebirgsgüte entwickelt, die unter dem $\mathrm{Na}$ men "Methoden zur Gebirgsklassifikation" bekannt sind. Diese Systeme sollen auf Basis von visueller Aufnahme des Gebirges in Kombination mit Laborversuchen Aufschluss über zu erwartendes Gebirgsverhalten geben. 
Die Gebirgsklassifikation ermöglicht eine geotechnische Einteilung des Gebirges in verschiedene Bewertungsklassen mit qualitativer Einteilung, wie beispielsweise "Sehr gutes Gebirge“, "Gutes Gebirge”, bis hin zu "Sehr schlechtes Gebirge." Daraus können in weiterer Folge beispielsweise Abschätzungen über die durchschnittliche Standzeit des Hohlraumes oder die erforderlichen Ausbaumaßnahmen gemacht werden. Um zu den im Bergbau erforderlichen quantitativen Bewertungen zu gelangen, wurden sodann Ansätze entwickelt, mit denen, resultierend aus den qualitativen Bewertungen, Parameter wie die Kohäsion, der Reibungswinkel, der Elastizitätsmodul oder die Festigkeit des Gebirges abgeschätzt werden können [1].

Folgende Methoden zur Gebirgsklassifikation werden in den meiste Fällen verwendet:

- Rock mass rating (RMR) nach Bieniawski (1989) [2]

- Mining rock mass rating (MRMR) nach Laubscher (1990) [3]

- Q-System nach Barton et al. (1974) [4]

- Geological strength index (GSI) nach Hoek (1994) [5]

Die erwähnten Methoden stellen den Anwender jedoch vor einige Schwierigkeiten, da jedes Verfahren für unterschiedliche Gebirgsverhältnisse entwickelt worden ist und die Anwendung der Verfahren nur bedingt für Gebirgstypen geeignet ist, die von den bei der Entwicklung berücksichtigten Umständen abweichen. Zusätzlich unterscheidet sich die heutige Anwendung zur Gebirgsgütebeurteilung teilweise vom Entstehungszweck der Systeme, welcher sich z. B. bei Laubscher auf die Eignung von Gebirge für Bruchbau bezog. In der vorliegenden Arbeit wird nun ein Überblick über die angeführten Klassifikationsverfahren und deren Inhalte gegeben, die generelle Problematik bei der Anwendung von Gebirgsklassifikation zur Gebirgsbeurteilung erläutert sowie konkrete Schwierigkeiten bei der Anwendung der einzelnen Methoden diskutiert. Abschließend wird ein handliches Tool präsentiert, mit welchem die Gebirgsaufnahmen für die betroffenen Systeme vereinfacht und übersichtlich gestaltet werden sollen, um somit die "Anwenderfreundlichkeit" zu erhöhen.

\section{Allgemeine Problematik der Gebirgsklas- sifikation}

Im Rahmen dieser Arbeit wurden die angeführten Klassifikationsverfahren in einem Untertagebergbau zur Beurteilung der Gebirgsgüte verwendet und im Zuge dessen die Schwierigkeiten und Probleme bei der Anwendung erörtert und diskutiert. Folgende kritische Punkte wurden bei der Anwendung aller Klassifikationsmethoden angetroffen:

- Subjektivität: Bewerten mehrere Personen denselben Gebirgsabschnitt, kommt es zu Abweichungen zwischen den einzelnen Ergebnissen. Dies liegt in erster Linie an der Schwierigkeit, an einer Felsoberfläche, die beispielsweise mittels konventionellen Vortriebes bzw. Abbaus geschaffen wurde, alle Diskontinuitäten richtig zu erkennen und einzuordnen.
Ein zusätzliches Problem tritt bei der Bestimmung einzelner Parameter auf, wie etwa die Oberflächenrauheit einer Kluftfläche, die nur qualitativ, unter anderem mit den Worten „sehr rau, , "rau“ oder „weich“ bewertet werden können und die Auffassung dieser Oberflächeneigenschaften von Person zu Person unterschiedlich ist. Die dadurch entstehenden Schwankungen der Ergebnisse von Gebirgsklassifikationen, welche von mehreren Personen im selben Abschnitt durchgeführt werden, konnte von Edelbro et al. (2006) in einer Studie belegt werden [6].

- Erfahrung (benötigt): Um zu einem aussagekräftigen Ergebnis zu gelangen, muss mit größter Sorgfalt und bester Kenntnis des Verfahrens an die Bestimmung der einzelnen Parameter herangegangen werden. Um gewisse Parameter richtig beurteilen zu können, ist Erfahrung bei der Anwendung von Gebirgsklassifikationssystemen unverzichtbar. Der Anwender sollte über ausreichende Kenntnis betreffend der Limitierungen der Anwendungsbereiche der einzelnen Systeme verfügen. Nur so können abweichende Ergebnisse durch subjektive und falsche Entscheidungen gering gehalten werden.

- Vergleich der unterschiedlichen Methoden: Vergleicht man die vier ausgewählten Methoden, so lässt sich feststellen, dass nur zwei Parameter in allen vier Verfahren zu finden sind, nämlich die Rauheit der Oberfläche einer Diskontinuität und der Verwitterungszustand. Weiters fällt bei den Eingangsgrößen eine unterschiedliche Gewichtung der einzelnen Faktoren für das Gesamtergebnis auf. Wie in den folgenden Kapiteln auch beschrieben wird, erfolgt die Berechnung der Gesamtbeurteilung auf unterschiedliche Art und Weise, was den Einfluss eines Eingangsparameters auf das Gesamtergebnis noch zusätzlich erhöht, welcher durch die unterschiedlichen Berechnungsmethoden bei jedem Verfahren stark variiert. Dies macht den Vergleich der einzelnen Methoden nahezu unmöglich, weswegen es sich empfiehlt, immer zumindest zwei unterschiedliche Methoden anzuwenden, um die Ergebnisse auf Plausibilität überprüfen zu können.

- Verfahrensabhängigkeit: Die einzelnen Methoden basieren auf Fallstudien verschiedener Bergbau- bzw. Tunnelbauprojekte und sind somit auf die dort gegebenen geologischen Eigenschaften ausgelegt. Es muss also bei der Auswahl der Verfahren genau beachtet werden, in welcher Geologie eine Gebirgsklassifikation angewendet wird, um kein verfälschtes Ergebnis zu erhalten, bzw. ob das jeweilige Verfahren für den gewünschten Anwendungszweck geeignet ist.

Die beschriebenen Punkte stellen grundsätzliche Hindernisse bei der Anwendung von Gebirgsklassifikation zur Gebirgsbeurteilung dar und treffen für alle Systeme gleichermaßen zu. In den nachstehenden Kapiteln wird auf die einzelnen Verfahren eingegangen und Schwierigkeiten bei ihrer Anwendung werden aufgezeigt. 


\section{Rock Mass Rating (RMR) nach Bieniawski (1989)}

Bieniawski (1973) erarbeitete mit dem Rock Mass Rating (RMR), auch als Geomechanics Classification bekannt, eine Methode, mit der anhand quantitativer Daten der benötigte Ausbau mittels Ankern und Spritzbeton abgeschätzt werden kann. Die Anwendungsbereiche erstrecken sich vom Tunnelbau und Felshängen über Festen bis hin zur Prüfung der Abtragbarkeit von Untergründen [2].

Um eine Gebirgsklassifikation mit dem RMR-System durchführen zu können, müssen folgende Parameter bestimmt werden [2]:

1. Festigkeit des intakten Gesteins

2. Rock quality designation (RQD)

3. Abstand der Diskontinuitäten zueinander

4. Zustand der Diskontinuitäten

5. Wasserverhältnisse

6. Orientierung der Diskontinuitäten

Das Endergebnis ergibt sich durch das Aufsummieren der Einzelratings der Punkte 1 bis 6 . In Summe kann ein maximaler Wert ("very good rock“) von 100 erreicht werden. Die Einteilung des Gebirges erfolgt in 5 Klassen von "Very good rock" bis "Very poor rock". Jede Klasse hat eine Bandbreite von jeweils 20 Wertungspunkten.

Jeder dieser sechs Punkte hat durch das Ratingsystem eine bestimmte Gewichtung auf das Endergebnis. Den größten Einfluss hat dabei der Zustand der Diskontinuitäten mit einem Ratingbereich von 0-30. Die Parameter mit den geringsten Einflüssen auf das Endergebnis sind die Festigkeit des intakten Gesteins, welche auf Basis des Point-Load-Index $\left(\mathrm{IS}_{50}\right)$ oder der einaxialen Druckfestigkeit (UCS) bestimmt wird, und die Wasserverhältnisse mit einer Bandbreite von 0-15.

Die Summe der ersten fünf Parameter wird Basis RMR genannt. Mit dem sechsten Parameter werden auch die Fallrichtung und der Fallwinkel der Diskontinuitäten in Bezug auf die Hohlraumausrichtung berücksichtigt (Bandbreite 0 bis -60). Dieser Parameter kann als einziger durch Anpassung der Hohlraumorientierung in Bezug auf signifikante Trennflächen beeinflusst werden. Unter Einbeziehung dieses Punktes wird in weiterer Folge das gesamte RMR gebil$\operatorname{det}[2]$.

Insbesondere bei den Eingangsparametern 3. und 4., die sich auf Diskontinuitäten und Klüfte beziehen, ergaben sich bei der untertägigen Anwendung einige Schwierigkeiten. Insbesondere bei der Bestimmung der Orientierung von Trennflächen, die in stark tektonisch beanspruchtem Gebirge häufig Orientierung und Verlauf ändern, konnte eine konservative Herangehensweise des Autors erkannt werden. An Diskontinuitäten, welche in ihren Verläufen und Ausrichtungen oft eine Veränderung aufweisen, muss die Orientierung in einem Messabschnitt an verschieden Stellen gemessen werden. Für das Rating der Orientierung wird die Messung mit dem größten Einfluss auf den Hohlraum verwendet. Somit liegt das Endergebnis auf der eher konservativen Seite.
Der Literatur konnte nicht entnommen werden, wie man zwei ineinander laufende Diskontinuitäten für das Rating des Abstandes der Diskontinuitäten zueinander bewertet. Um wiederum ein eher konservatives Ergebnis zu erreichen, wurde in diesem Fall mit dem geringsten Ratingwert gerechnet.

\section{Mining Rock Mass Rating (MRMR) nach Laubscher (1990)}

Das Mining Rock Mass Rating wurde 1974 von Laubscher als Weiterentwicklung des Rock Mass Rating Systems nach Bieniawski speziell für den Anwendungsbereich im Bergbau, genauer gesagt für den Blockbruchbau entwickelt. Wie auch andere Verfahren wurde dieses über die Jahre mehrmals überarbeitet und weiterentwickelt. In diesem Artikel wird Bezug auf die Version von 1990 genommen.

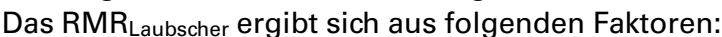

1. Rating für die durchschnittliche Festigkeit des intakten Gesteins (RIRS)

2. Rating des ROD-Wertes ( $\left.R_{\text {ROD }}\right)$

3. Rating der Kluftabstände (RJs)

4. Rating des Zustandes der Klüfte und der Wasserverhältnisse ( $\left.R_{J C}\right)$

Dabei ist zu beachten, dass das $R M R_{\text {Laubscher nicht dem }}$

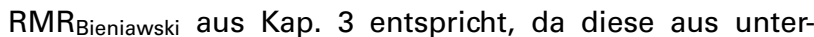
schiedlichen Einflussfaktoren und unterschiedlichen Gewichtungen entstehen.

Zu berücksichtigen ist, dass es sich beim $\mathrm{R}_{\mathrm{IRS}}$-Wert nicht um die einaxiale Druckfestigkeit (UCS) handelt, sondern um eine von Laubscher entwickelte durchschnittliche Festigkeit des intakten Gesteins. Für das Rating der durchschnittlichen Festigkeit des intakten Gesteins ( $R_{\mathrm{IRS}}$ ) muss zuerst die einaxiale Druckfestigkeit des festen und schwächsten Gesteins bestimmt werden. Erst danach kann mittels desDiagramms von Abb. 1 die benötigte durchschnittliche Festigkeit ermittelt werden [3]. Dies ist grundsätzlich nur bei sedimentären Lagerstätten zielführend und sollte bei nicht sedimentären Lagerstätten nicht berücksichtigt werden. Im Falle von nicht sedimentären Lagerstätten sollte auf die Bestimmung einer durchschnittlichen Festigkeit verzichtet werden und auf die einaxiale Druckfestigkeit des Materials zurückgegriffen werden.

Die Ratings des ROD-Wertes ( $\mathrm{R}_{\mathrm{ROD}}$ ) und der Kluftabstände (RJs) können durch das Fracture Frequency per Metre (FF/m) ersetzt werden. Dieser Wert ergibt sich aus dem Verhältnis der Anzahl aller Diskontinuitäten entlang einer Messstrecke zur Länge der Messstrecke in Meter. Bei der Bestimmung des Fracture Frequency per Metre (FF/m) wird jedoch nur zwischen Systemen mit einer, zwei oder drei Kluftscharen unterschieden.

Der Wert des FF/m muss zusätzlich mit einem Faktor korrigiert werden, welcher anhand einer Tabelle bestimmt wird und davon abhängig ist, an welchen Stellen (Ulme, Firste, oder Ulme und Firste) die Diskontinuitäten eines Abschnittes gemessen werden. Diese Tabelle ist schwer anzuwenden und erhöht den Zeitaufwand des Verfahrens erheblich. 


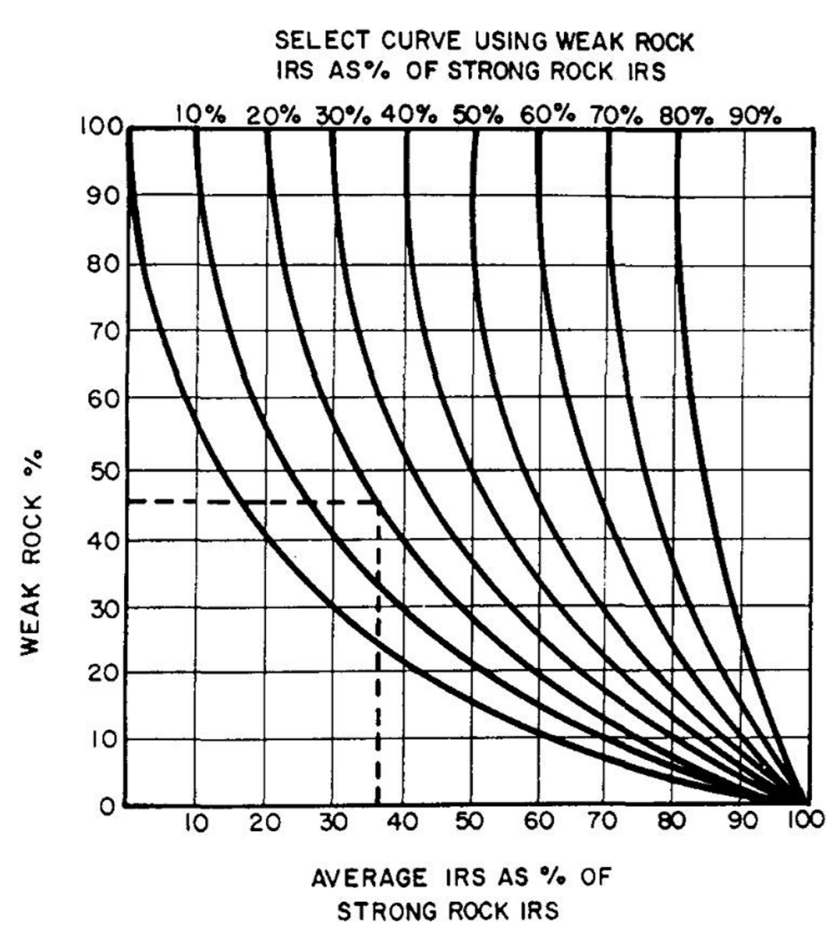

Abb. 1: Diagramm zur Bestimmung der IRS [2]

Der Wert für das Rating der Kluftabstände (RJs) wird mittels Formeln berechnet, welche Taylor zur Ersetzung des ursprünglichen Charts entwickelte [3]. Diese Formeln sind jedoch nur für Anwendungsfälle mit ein, zwei, oder drei vorliegenden Kluftscharen anwendbar. Die Bewertung dieses Parameters auf Abschnitte ohne erkennbare Kluftscharen wird nicht angegeben.

Besonders kompliziert in der Anwendung erwiesen sich auch die Anpassungsfaktoren für die Kluftorientierung und Spannungsumlagerung. Da bei diesen der zeitliche Aufwand zur Anwendung den Nutzen deutlich übersteigt, wurden diese im ausgearbeiteten Worksheet nicht berücksichtigt.

Die Anwendung des Klassifikationsverfahrens nach Laubscher ist sowohl zeitaufwändig als auch kompliziert. Dies konnte auch von Edelbro et al. (2006) anhand einer Studie belegt werden, wo auf die große Anzahl an Tabellen und schwierig anzuwendende Anpassungsfaktoren hingewiesen wird [6].

\section{Q-System nach Barton et al. (1974)}

Das Q-System wurde 1974 in Norwegen von Barton, Lien und Hunde entwickelt. Das Verfahren basiert auf 212 Fallstudien aus dem Tunnelbau in Skandinavien. Es ist ein quantitatives Klassifikationssystem und stellt eine Hilfe zur Ausbaufestlegung dar [4].

Der Großteil der Fallstudien, auf denen dieses Verfahren basiert, wurde in hartem, geklüftetem Gebirge durchgeführt. Nur wenige dieser Studien entstanden in wenig tektonisch beanspruchtem Gebirge. Daher sollten in diesem Fall zusätzlich andere Systeme der Gebirgsklassifikation für die Ausbauplanung angewendet werden [7].

Das Q-System setzt sich aus folgenden sechs zu bestimmenden Parametern zusammen:

1. Rock quality designation (ROD)

2. Anzahl der Kluftscharen $\left(J_{n}\right)$

3. Rauheit der Kluft $\left(J_{r}\right)$

4. Verwitterung der Kluft $\left(J_{a}\right)$

5. Wassereigenschaften $\left(J_{w}\right)$

6. Spannungszustand (SRF)

Gepaart beschreiben diese Parameter die drei Hauptfaktoren, welche die Stabilität des Gebirges beeinflussen:

- Der Quotient der ersten beiden Parameter repräsentiert die Gesamtstruktur bzw. die Blockgröße des ungestörten Gebirges.

- Der Quotient des dritten und vierten Parameters beschreibt die Scherfestigkeit der Klüfte.

- Der Quotient des fünften und sechsten Parameters ergibt den lokalen Spannungszustand.

[4].

Der Q-Wert ergibt sich folgendermaßen:

$$
Q=\frac{\mathrm{RQD}}{J_{n}} \times \frac{J_{r}}{J_{a}} \times \frac{J_{w}}{\mathrm{SRF}}
$$

Er liegt in einer Bandbreite von 0,001 bis 1000 auf einer logarithmischen Skala und unterscheidet sich somit von den bereits besprochenen Systemen, deren Bewertung auf Basis einer Skala von 0-100 erfolgt.

Die qualitative Beschreibung des Gebirges und die vorgeschlagene Ausbaumethode können anhand des Diagramms in Abb. 2 bestimmt werden.

Hier konnten keine speziellen Anwendungsprobleme aufgezeigt werden. Es gelten die allgemeinen Schwierigkeiten bei der Bestimmung der Rauheit und Verwitterung von den Diskontinuitäten.

\section{Geological Strength Index (GSI) nach Hoek (2002)}

Der Geological Strength Index (GSI) nach Hoek (2002) ist eine der jüngsten Anwendungsmethoden zur Klassifizierung von Gebirge. Im Gegensatz zum RMR- oder Q-System wurde das GSI-System nicht zur Abschätzung der benötigten Ausbaumaßnahmen entwickelt, sondern um die Eigenschaften (Festigkeit und Elastizitätsmodul) des Gebirges bestimmen zu können [5].

Dieses Verfahren basiert auf Charts, welche einige Male weiterentwickelt wurden.

Die Version von Hoek und Marinos (2000) teilt das Chart auf der $y$-Achse in sechs unterschiedliche Klassen der Gebirgsstruktur (intaktes bis laminiertes Gebirge) ein. Die $x$-Achse wird in fünf Klassen der Rauheit und des Verwitterungszustandes der Diskontinuitäten eingeteilt. Dieses Verfahren basiert somit auf einer rein visuellen Bewertung des Gebirges. 


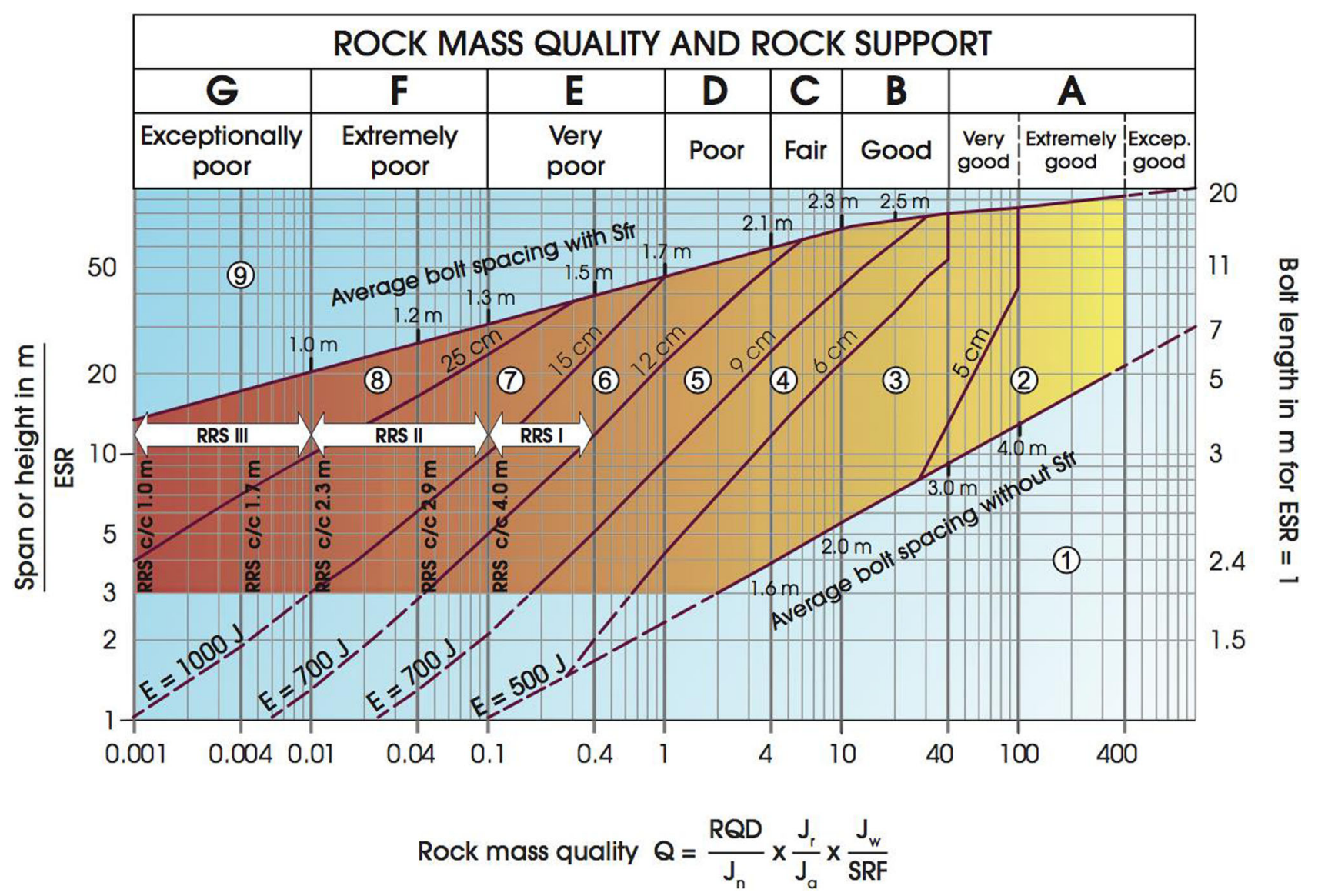

Abb. 2: Ausbauempfehlung nach Barton [7]

Um eine objektivere Bewertung des GSI-Systems zu gewährleisten, wurde ein Chart mit quantitativen Input-Parametern erstellt. Die $x$-Achse wurde um den Joint Condition Factor (Jc) und die y-Achse um das Block Volume (Vb) erweitert.

Der Joint Condition Factor (Jc) wird aus drei Parametern berechnet:

$$
J_{C}=J_{W} \frac{J_{S}}{J_{A}}
$$

$J_{W}$ beschreibt die großflächige Unebenheit der Diskontinuität, $\mathrm{J}_{\mathrm{S}}$ die kleinflächigen Unebenheiten und $\mathrm{J}_{\mathrm{A}}$ den Verwitterungszustand.

Für detaillierte Studien zur Berechnung und Anwendung des Joint Condition Factors und Block Volumes wird die Literatur von Palmström A. (2001) empfohlen [8].

Weiters wurde ein Chart entworfen, welches mit den Ratingwerten des RMR-Systems nach Bieniawski (1989) arbeitet. Auf der x-Achse wird der Ratingwert des Zustandes der Diskontinuität mit 1,5 multipliziert und auf der y-Achse der Ratingwert des ROD durch 2 dividiert. Anhand dieser Werte kann, ohne Bestimmung zusätzlicher Parameter, der GSIFaktor im Chart ermittelt werden.

Den größten Aufwand für die Bestimmung des GSI-Faktors verursacht jenes Chart, bei dem der Joint Condition Factor ( Jc) und das Block Volume (Vb) benötigt werden.
Um den Joint Condition Factor berechnen zu können, erfordert es zuvor eine gründliche und zeitaufwändige Aufnahme der erwähnten Parameter. Um beispielsweise den $\mathrm{J}_{\mathrm{W}}$-Wert bestimmen zu können, muss die maximale Amplitude der Diskontinuität gemessen werden. Dies erfolgt durch das Anlegen einer 0,9 m langen Latte an die Gebirgsoberfläche. Das Anlegen der von Piteau (1970) vorgeschlagenen 0,9 m langen Latte ist jedoch aus gebirgstechnischen Gegebenheiten nicht immer möglich und muss daher angepasst werden. Um entlang einer Diskontinuität die maximale Auslenkung bestimmen zu können, müsste somit die gesamte Länge beurteilt werden.

Daraus ergibt sich, dass der Aufwand zur Anwendung dieses Charts nicht in Relation mit dem damit verbundenen Nutzen steht.

\section{Arbeitsblatt zur einfachen Anwendung der Gebirgsklassifikationssysteme}

Für die Ausarbeitung des erwähnten Tools zur Vereinfachung der Gebirgsaufnahmen musste zunächst ein Überblick über die benötigten Eingangsparameter und die Übereinstimmungen der einzelnen Gebirgsklassifikationen geschaffen werden. Im Anschluss daran konnte ein erstes Worksheet in Tabellenform angefertigt werden (Abb. 3). Es wurde versucht, die Reihung der zu bestimmenden 


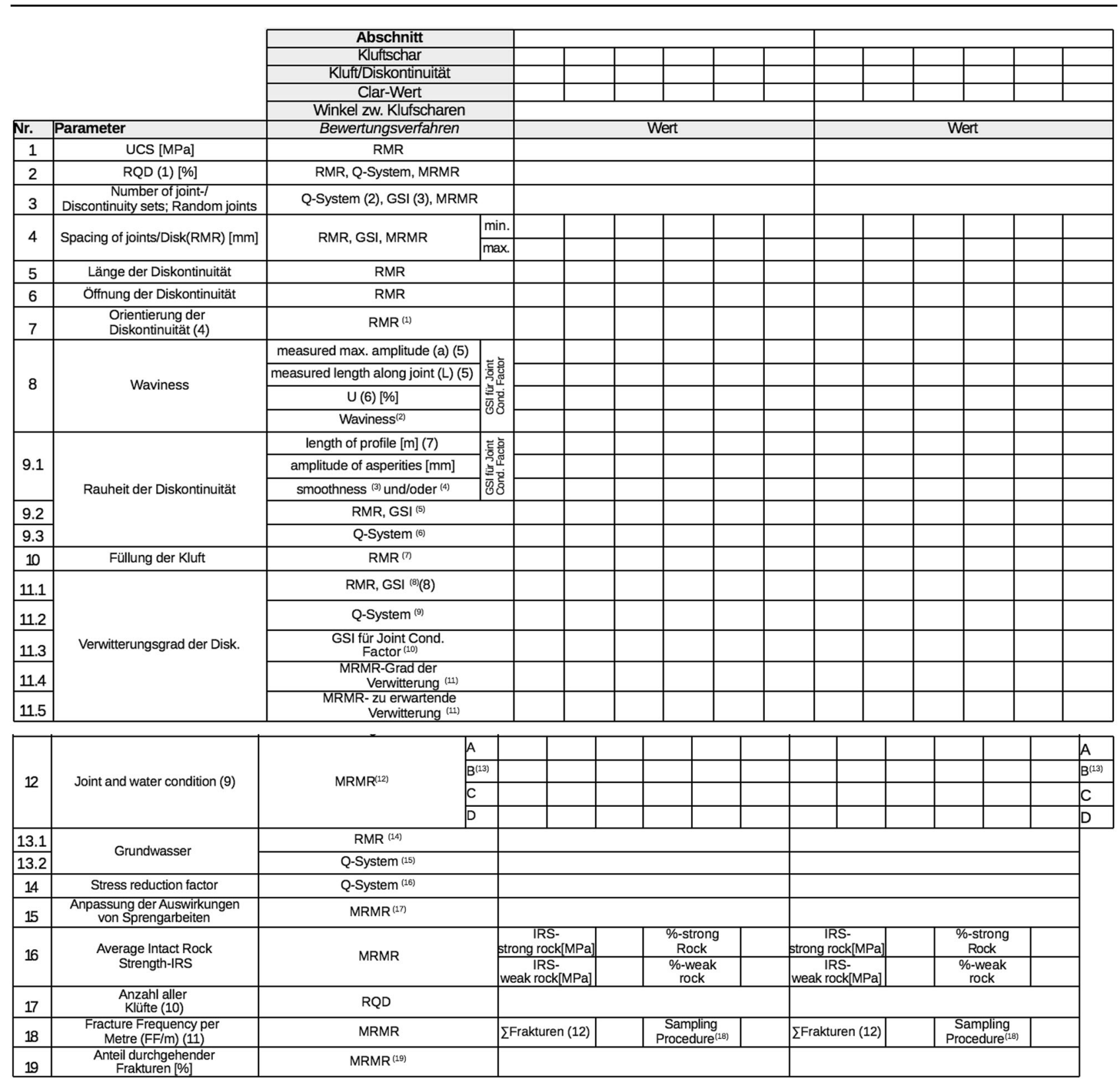

Skizze Abschnitt:

Skizze Abschnitt:
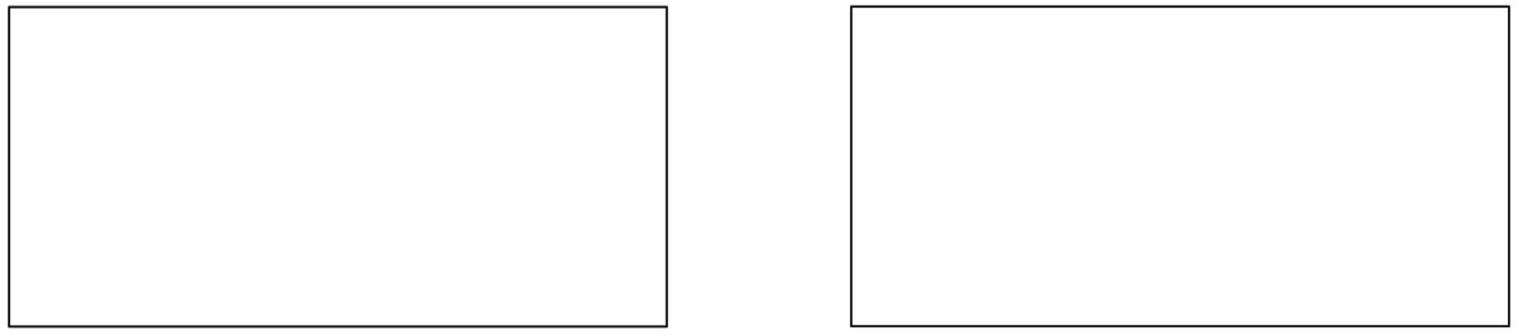

Abb. 3: Arbeitsblatt zur vereinfachten Anwendung von Gebirgsklassifikationssystemen 
Parameter in der Tabelle so zu gestalten, dass ein flüssiger Arbeitsablauf während der Aufnahme gewährleistet wird. Pro Messabschnitt wurden sechs Spalten erstellt, von welchen jede einzelne für die Bewertung einer Diskontinuität zur Verfügung steht. Dies ermöglicht, für jeden Messabschnitt die Diskontinuität mit dem größten Einfluss auf das Gebirge zu ermitteln. Durch das mehrmalige Anwenden des Tools in einem untertägigen Bergbau konnten fehlende und überflüssige Parameter weiter angepasst werden. Die hochgestellten Nummerierungen verweisen auf Tabellen der jeweiligen Bewertungssysteme, wodurch eine Bewertung der einzelnen Parameter durchgeführt werden kann. Um das Arbeitsblatt bezüglich seiner Nutzerfreundlichkeit weiter zu optimieren, bedarf es noch weiterer Überarbeitung. Dennoch stellt dieses Tool eine Möglichkeit der Gebirgsaufnahme für vier verschiedene Systeme der Gebirgsklassifikation dar, welches die Verwendung weiterer Unterlagen während der Anwendung obsolet macht.

\section{Schlussfolgerung}

Im Rahmen der vorliegenden Arbeit wurden die geläufigsten Methoden zur Gebirgsklassifikation diskutiert, analysiert und grundlegende Anwendungsschwierigkeiten und Probleme identifiziert. Auf Basis der mehrfachen Anwendung der Systeme im Untertagebergbau wurde in weiterer Folge ein Arbeitsblatt zur Vereinfachung der Anwendung von Gebirgsklassifikation als Mittel zur Gebirgsgütebeschreibung entwickelt.

Aus den durchgeführten Analysen kann geschlossen werden, dass sich sowohl das "Mining Rock Mass Rating" (MRMR) nach Laubscher als auch das Chart des "GSISystems" nach Hoek, welches den Joint Condition Factor und das Block Volume zur Bestimmung verwendet, in ihrer Anwendung als besonders aufwändig und kompliziert darstellen. Im Falle des MRMR-Systems kommen einige komplizierte Tabellen und Anpassungsfaktoren zum Einsatz. Die hohe Anzahl dieser Anpassungsfaktoren führt zu einem erheblich verschlechterten Endrating und somit auch zu einem sehr konservativen Ergebnis der Gebirgsklassifikation. Im Falle des GSI-Systems in der Version, die das Blockvolumen und den Joint Condition Factor verwendet, sind es ebenfalls viele zusätzlich zu bestimmende Einflussparameter, welche die Anwendung dieser Methode maßgeblich erschweren.

Durch ihre klar strukturierten Tabellen und verhältnismäBig einfach zu bestimmenden Aufnahmeparameter haben sich die Systeme von Bieniawski (RMR) und Barton (Q) als besonders anwenderfreundlich erwiesen. Diese zwei Systeme sind leicht anzuwenden, liefern jedoch aufgrund ihrer Einfachheit einige Simplifizierungen bezogen auf die $\mathrm{Ge}-$ birgsbeurteilung.

Durch das erarbeitete Worksheet kann die Effizienz des Arbeitsvorganges zur vereinfachten Aufnahme des Gebirges gesteigert werden. Im Zuge der Erarbeitung des Worksheets konnten auch eventuell auftretende Schwierigkeiten in der Gebirgsaufnahme bezüglich der jeweiligen Systeme der Gebirgsklassifikation aufgezeigt sowie die Anwendbarkeit der einzelnen Methoden einer kritischen Betrachtung unterzogen werden. Grundvoraussetzung einer erfolgreichen, insbesondere korrekten Ausführung sind für den Anwender jedoch immer Kenntnis und Erfahrung im Bereich der unterschiedlichen Verfahren. Nur so können Fehler bei der Interpretation der Parameter vermieden und der dazugehörige Zeitaufwand möglichst gering gehalten werden. An dieser Stelle soll noch angemerkt werden, dass dieses Tool anhand von Aufnahmen in einem Untertagebergbau entwickelt und entsprechend den dortigen Gegebenheiten angepasst wurde. Daher sollte dieses Arbeitsblatt auch anhand von Aufnahmen in anderen Bergbaubetrieben getestet und eventuell überarbeitet werden. Weiters sollte beachtet werden, dass die einzelnen Systeme teilweise vereinfacht und angepasst wurden, um eine möglichst unkomplizierte Arbeitsvorlage zu schaffen. In weiterer Folge sollte noch geklärt werden, inwieweit diese Vereinfachungen das Endergebnis beeinflussen. Das beschriebene Arbeitsblatt wurde vom Autor dieser Arbeit zusammengestellt. Zukünftig sollte es von unterschiedlichen Personen angewandt werden, um zu überprüfen, ob die Vergleichbarkeit der Ergebnisse verschiedener Personen mit dem erarbeiteten Tool gesteigert werden konnte.

Open access funding provided by Montanuniversität Leoben.

Open Access Dieser Artikel wird unter der Creative Commons Namensnennung 4.0 International Lizenz (http://creativecommons.org/licenses/ by/4.0/deed.de) veröffentlicht, welche die Nutzung, Vervielfältigung, Bearbeitung, Verbreitung und Wiedergabe in jeglichem Medium und Format erlaubt, sofern Sie den/die ursprünglichen Autor(en) und die Quelle ordnungsgemäß nennen, einen Link zur Creative Commons Lizenz beifügen und angeben, ob Änderungen vorgenommen wurden.

\section{Literatur}

1. Siefert, M.: Verfahren zur qualitativen und quantitativen Beschreibung des Gebirges. 1.Teil: Qualitative Gebirgsbeschreibung, BHM Berg- und Hüttenmännische Monatshefte 154 (2009), S. 272-286

2. Bieniawski, Z.T.: Engineering rock mass classification: a complete manual for engineers and geologists in mining, civil, and petroleum engineering, JWiley, 1989

3. Laubscher, D.H.: A geomechanics classification system for the rating of rock mass in mine design, Journal of the South African Institute of Mining and Metallurgy, vol. 90 (1990), no. 10, pp. 257-273

4. Barton, N.: Scale effects or sampling bias?, in: A. Pinto da Cunha (ed), Proceedings of the First International Workshop on Scale Effects in Rock Masses, 7-8 June, Loen, Norway, Rotterdam: Balkema, 1990, pp. 31-59

5. Hoek, E.; Carranza-Torres, C.: Corkum, B.: Hoek-Brown failure criterion - 2002 edition, in: R. Hammah (ed), Proceedings of the 5th North American Rock Mechanics Symposium and the 17th Tunnelling Association of Canada Conference, University of Toronto, Toronto, Ontario, pp. 267-273

6. Edelbro, C.; Sjöberg, J.; Nordlund, E.: A quantitative comparison of strength criteria for hard rock masses, Tunneling and Underground Space Technology, 22 (2007), Issue 1, p. 57-68

7. NGI: Using the Q-system. Rock mass classification and support design, NGI, 2013

8. Palmström, A.: In-Situ Characterization of rocks. Chapter 2: Measurement and characterization of rock mass jointing, Lise, Abingdon, Exton (PA), Tokio: Balkema, 2001 